\title{
Improving access to urgent rheumatology - the initiation of a hot joint assessment and injection service
}

\author{
Authors: Jennifer Christie and Katherine Nelson
}

\begin{abstract}
Aims
To improve the joint injection service provided locally while providing rapid access outpatient appointments for patients admitted to hospital with acute rheumatological presentations.

\section{Methods}

We identified a need to improve the joint injection service as there was only one dedicated injection clinic per week that provided a limited number of joint injections for acutely swollen joints. Patients would often wait over a week with an acutely swollen, painful joint. We felt this could be improved and set up an additional 'hot joint assessment clinic'. This clinic was also used for rapid access to rheumatology services which facilitated and expedited inpatient discharges while also reviewing urgent primary care referrals. We used an online synchronised Outlook calendar to populate the list of patients which was advertised by email to the entire rheumatology department. As part of this innovative service, the injection consent pro forma was digitalised and made available on the intranet to further standardise the documentation of intra-articular injections within our trust. Finally as part of our service, a 'one-stop injection trolley' was devised which included all the equipment and documentation necessary for efficient and effective intra-articular injections.
\end{abstract}

\section{Results}

We have seen 70 patients since September 2017 in the "hot joint assessment clinic'. This has included several primary care patients that were seen in lieu of a routine outpatient appointment due to rapidly deteriorating symptoms or a possible life-threatening diagnosis, such as vasculitis. We have also reviewed patients with swollen joints not yet referred to ourselves that the primary care team have been unable to manage. By referring the patients straight to our service we have reduced referrals to $A \& E$ and the acute medical unit. We have been able to reduce length of hospital stay of patients with joint problems by providing rapid follow-up.

Authors: Rheumatology, Royal Liverpool and Broadgreen University Hospitals NHS Trust, Liverpool, UK

\section{Conclusion}

We feel proud that this service is continuing to meet its objectives and runs on a weekly basis. The steroid injection consent process is now standardised across the trust and patients no longer wait more than 1 week for treatment for an acutely swollen joint.

Most hospital inpatients with rheumatology problems can be safely discharged with timely follow-up, reducing inpatient stay and further optimising the use of acute medical beds.

\section{Conflict of interest statement}

None declared. 